\title{
Study of the Antarctic Circumpolar Current via the Shallow Water Large Scale Modelling
}

\author{
Kateryna Marynets
}

Faculty of Mathematics, University of Vienna, Oskar-Morgenstern-Platz 1, 1090 Vienna, Austria

kateryna.marynets@univie.ac.at

Keywords: Geophysical Flow, Nonlinear Boundary-Value Problem, Global Existence, Uniqueness, Positive-Definite Function

\begin{abstract}
This paper proposes a modelling of the Antarctic Circumpolar Current (ACC) by means of a two-point boundary value problem. As the major means of exchange of water between the great ocean basins (Atlantic, Pacific and Indian), the ACC plays a highly important role in the global climate. Despite its importance, it remains one of the most poorly understood components of global ocean circulation. We present some recent results on the existence and uniqueness of solutions of a two-point nonlinear boundary value problem that arises in the modeling of the flow of the (ACC) (see discussions in [4-9]).
\end{abstract}

\section{Introduction}

The Antarctic Circumpolar current is one of the five main currents. It is the only current that flows completely around the globe, and is the strongest and largest wind-driven ocean current on the planet. It extends from the bottom of the ocean to the sea surface and is the primary means of inter-basin exchange.

The flow of ACC can be modelled using the following two-point boundary-value problem

$$
\left\{\begin{array}{l}
u^{\prime \prime}(t)=a(t) F(u(t))-b(t), \quad t \in(0,1) \\
u(0)=u_{0}, \quad u\left(t^{*}\right)=u^{*}
\end{array}\right.
$$

where $F: R \rightarrow R$ is a given continuous function and $a, b:[0,1) \rightarrow[0,1)$ are given bounded continuous functions satisfying

$$
\int_{0}^{1} a(s)+b(s) d s<\infty
$$

was recently derived as a model for the azimuthal horizontal jet flow components of the Antarctic Circumpolar Current. The existence of nontrivial solutions is of considerable interest, since these correspond to azimuthal flows that feature variations in the meridional direction, being thus models that capture the essential geophysical features, confirmed by field data.

A general result ensures the existence and uniqueness of a solution to Eq. 1, provided that for every $\varepsilon \in(0,1)$ we have:

- all solutions of the initial-value problem 
$\left\{\begin{array}{l}u^{\prime \prime}(t)=a(t) F(u(t))-b(t), \quad t \in(0,1), \\ u(0)=u_{0}, \\ u^{\prime}(0)=u_{1},\end{array}\right.$

exist on $[0,1+\varepsilon)$ for all $u_{0}, u_{1} \in \mathrm{R}$;

- there do not exist two solutions on $\left[0, t^{*}\right]$ to the two-point boundary-value problem

$\left\{\begin{array}{l}u^{\prime \prime}(t)=a(t) F(u(t))-b(t), \quad t \in(0,1), \\ u(0)=u_{0}, \quad u\left(t^{*}\right)=u^{*},\end{array}\right.$

for any $t^{*} \in(1-\varepsilon, 1+\varepsilon)$ and $u^{*} \in \mathrm{R}$.

In particular, global existence for Eq. 2 and uniqueness for Eq. 3 ensure the solvability of Eq. 1. While this is by no means the only possible approach that can accommodate nonlinear functions $F$, it has some advantages over more classical methods in that the type of hypotheses that need to be verified seem to accommodate large classes of functions.

\section{Modeling of the ACC}

For terrestrial regions outside the poles let us introduce the spherical coordinates (see Fig.1):

- $\theta \in[0, \pi)$ is the polar angle (with $\theta=0$ corresponding to the North Pole and with $\theta=\frac{\pi}{2}$ along the Equator);

- $\varphi \in[0,2 \pi)$ is the angle of longitude (or azimuthal angle).

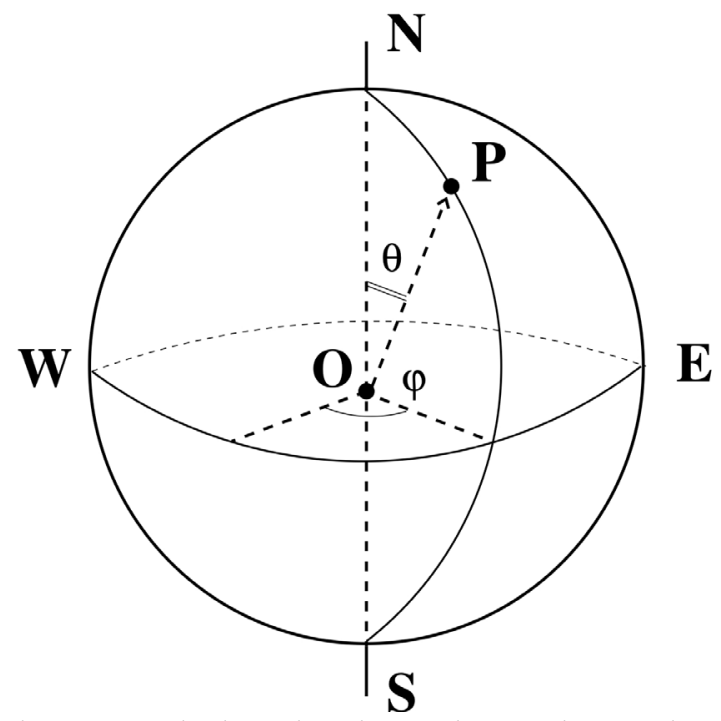

Figure 1: Depiction of the azimuthal and polar spherical coordinates of a point $P$ on the spherical surface of the Earth:

In terms of the stream function $\psi(\theta, \varphi)$, a horizontal ocean flow on the spherical Earth has azimuthal and meridional velocity components given by $(\csc \theta) \psi_{\phi}$ and $-\psi_{\theta}$; see [3]. 
By associating $\Psi(\theta, \varphi)$ with the vorticity of the ocean motion (not accounting for the effects of the Earth's rotation), given by

$$
\psi(\theta, \varphi)=-\omega \cos \theta+\Psi(\theta, \varphi)
$$

where $\omega>0$ is the non-dimensional form of the Coriolis parameter, the governing equation for the horizontal flow on the sphere takes the form (see [2])

$$
\frac{1}{\sin ^{2} \theta} \Psi_{\varphi \varphi}+\Psi_{\theta} \cot \theta+\Psi_{\theta \theta}=F(\Psi-\omega \cos \theta)
$$

where $F(\Psi-\omega \cos \theta)$ is the oceanic vorticity, while $2 \omega \cos \theta$ is the planetary vorticity, generated by the Earth's rotation.

By means of the stereographic projection

$$
\xi=r e^{i \phi} \quad \text { with } \quad r=\cot \left(\frac{\theta}{2}\right)=\frac{\sin \theta}{1-\cos \theta}
$$

where $(r, \phi)$ are the polar coordinates in the equatorial plane, we transform the model Eq. 5 in spherical coordinates into an equivalent planar elliptic partial differential equation.

More precisely, using the complex variable $\xi$, we can write Eq. 5 as

$$
\psi_{\xi \bar{\xi}}+2 \omega \frac{1-\xi \bar{\xi}}{(1+\xi \bar{\xi})^{3}}-\frac{F(\psi)}{(1+\xi \bar{\xi})^{2}}=0 .
$$

Using the Cartesian coordinates $(x, y)$ in the complex $\xi$-plane, the equation Eq. 7 is equivalent to the following semilinear elliptic partial differential equation

$$
\Delta \psi+8 \omega \frac{1-\left(x^{2}+y^{2}\right)}{\left(1+x^{2}+y^{2}\right)^{3}}-\frac{4 F(\psi)}{\left(1+x^{2}+y^{2}\right)^{2}}=0,
$$

where $\Delta=\partial_{x}^{2}+\partial_{y}^{2}$ denotes the Laplace operator; see [3, 4].

Since the ACC presents a considerable uniformity in the azimuthal direction (see the discussions in $[1,4]$ ), we can take advantage of this feature to simplify the problem Eq. 8 further.

Indeed, solutions with no variation in the azimuthal direction correspond to radially symmetric solutions $\psi=\psi(r)$ of the problem Eq. 8 .

The change of variables

$$
\psi(r)=U(s), \quad s_{1}<s<s_{2},
$$

with $r=e^{-s / 2}$ for $0<s_{1}=-2 \ln \left(r_{+}\right)<s_{2}=-2 \ln \left(r_{-}\right)$, for $0<r_{-}<r_{+}<1$, can now be used to transform the partial differential equation Eq. 8 to the second-order ordinary differential equation 


$$
U^{\prime \prime}(s)-\frac{e^{s}}{\left(1+e^{s}\right)^{2}} F(U(s))+\frac{2 \omega e^{s}\left(1-e^{s}\right)}{\left(1+e^{s}\right)^{3}}=0, s_{1}<s<s_{2} .
$$

Note that, for $0<s_{1}<s_{2}$, the change of variables

$$
u(t)=U(s) \quad \text { with } t=\frac{s-s_{1}}{s_{2}-s_{1}}
$$

transforms the second-order differential equation Eq. 10 to the equivalent one of the form

$$
u^{\prime \prime}=a(t) F(u)-b(t), \quad 0<t<1,
$$

where $a(t)=\frac{e^{t}}{\left(1+e^{t}\right)^{2}}, \quad b(t)=\frac{2 \omega e^{t}\left(1-e^{t}\right)}{\left(1+e^{t}\right)^{3}}$.

We will couple the derived differential equation with the Dirichlet conditions on the boundary $\partial \mathrm{D}$ of $\mathrm{D}$

$$
u\left(t_{1}\right)=u_{0}, u\left(t_{2}\right)=\alpha
$$

which reflect the physically relevant condition that $D$ represents the stereographic projection of a surface on the sphere delimited by two streamlines.

\section{Main results}

The following result provides sufficient conditions for the global existence of the solutions to the initial-value problem Eq. 2.

Theorem 1. [8] If the continuous function $F: \mathrm{R} \rightarrow \mathrm{R}$ satisfies

$$
M+\int_{0}^{u} F(\xi) d \xi \geq W^{-1}\left(F^{2}(u)\right), \quad u \in \mathrm{R},
$$

for some constant $M>0$ and some strictly increasing function $W:[0, \infty) \rightarrow[0, \infty)$ with $W(0)=0, W(s)>0$ for $s>0$ and satisfying

$$
\int_{1}^{\infty} \frac{d u}{W(u)}=\infty
$$

and if

$$
\lim _{|u| \rightarrow \infty} \int_{0}^{u} F(\xi) d \xi=\infty
$$

then all solutions of (2) are global in time. 
Since Theorem 1 proves the global existence of solutions of the initial-value problem Eq. 2, we now study the question of the uniqueness of solutions to the two-point boundary-value problem Eq. 1.

Theorem 2. [8] If the continuous function $F: \mathrm{R} \rightarrow \mathrm{R}$ is monotone nondecreasing on $\mathrm{R}$, then the solution of the Eq. 3 is unique.

Theorem 3, [8] Assume that the continuous function $F: \mathrm{R} \rightarrow \mathrm{R}$ is monotone nondecreasing and satisfies the conditions Eq. 14 and Eq.16, for some nondecreasing continuous function $W:[0, \infty) \rightarrow[0, \infty)$ with $W(0)=0, W(u)>0$ for $u>0$, and subject to the constraint Eq. 15. Then the problem Eq. 1 admits a unique solution.

\section{Acknowledgements}

The support of the WWTF grant MA16-009 is gratefully acknowledged.

\section{References}

[1] A. Constantin and R. S. Johnson, An exact, steady, purely azimuthal flow as a model for the Antarctic Circumpolar Current, J. Phys. Oceanography, 46 (2016) 3585-3594.

https://doi.org/10.1175/JPO-D-16-0121.1

[2] A. Constantin and R. S. Johnson, Large gyres as a shallow-water asymptotic solution of Euler's equation in spherical coordinates, Proc. Roy. Soc. London A, 473 (2017), Art. 20170063 , 17 pp. https://doi.org/10.1098/rspa.2017.0063

[3] A. Constantin and R. S. Johnson, Steady large-scale ocean flows in spherical coordinates, Oceanography, 31 (2018), 42-50. https://doi.org/10.5670/oceanog.2018.308

[4] S. V. Haziot and K. Marynets, Applying the stereographic projection to the modeling of the flow of the Antarctic Circumpolar Current, Oceanography, 31 (2018), 68-75.

https://doi.org/10.5670/oceanog.2018.311

[5] K. Marynets, On a two-point boundary-value problem in geophysics, Applicable Analysis, 98 (2019), 553-560. https://doi.org/10.1080/00036811.2017.1395869

[6] K. Marynets, A nonlinear two-point boundary-value problem in geophysics, Monatsh Math., 188 (2019), 287-295. https://doi.org/10.1007/s00605-017-1127-x

[7] K. Marynets, Two-point boundary-value problem for modeling the jet flow of the Antarctic Circumpolar Current, Electronic J. Diff. Eq., 56 (2018), 12 pp.

[8] Kateryna Marynets, Study of a nonlinear boundary-value problem of geophysical relevance, Discrete and Continuous Dynamical Systems (2019), 39(8), 4771-4781.

https://doi.org/10.3934/dcds.2019194

[9] Kateryna Marynets, The Antarctic Circumpolar Current as a shallow-water asymptotic solution of Euler's equation in spherical coordinates, Deep-Sea Research Part II (2019), (160), 58-62. https://doi.org/10.1016/j.dsr2.2018.11.014 